\title{
BMJ
}

\section{Elbow extension test to rule out elbow fracture: multicentre, prospective validation and observational study of diagnostic accuracy in adults and children}

\begin{abstract}
A Appelboam, consultant emergency physician, ${ }^{1}$ A D Reuben, consultant emergency physician, ${ }^{1}$ J R Benger, consultant emergency physician, ${ }^{2} \mathrm{~F}$ Beech, specialist registrar, ${ }^{3}$ J Dutson, specialist registrar, ${ }^{4} \mathrm{~S}$ Haig, specialist registrar, ${ }^{3}$ I Higginson, consultant emergency physician, ${ }^{2}$ I A Klein, specialist registrar, ${ }^{5}$ S Le Roux, specialist registrar, ${ }^{6}$ S S M Saranga, specialist registrar, ${ }^{6}$ R Taylor, consultant emergency physician, ${ }^{1}$ J Vickery, emergency nurse practitioner, ${ }^{1}$ R J Powell, statistician, ${ }^{7} \mathrm{G}$ Lloyd, consultant emergency physician ${ }^{1}$
\end{abstract}

'Emergency Department, Royal Devon and Exeter Foundation NHS Trust, Exeter EX2 5DW

2Emergency Department, United Bristol Healthcare NHS Trust, Bristol BS2 8HW

${ }^{3}$ Emergency Department, Bath Royal United Hospital NHS Trust, Bath BA1 3NG

${ }^{4}$ Emergency Department, Bristol Royal Infirmary, United Bristol Healthcare NHS Trust, Bristol BS1

${ }^{5}$ Emergency Department,

Musgrove Park Hospital, Taunton and Somerset NHS Trust, Taunton TA1 5DA

${ }^{6}$ Emergency Department, Bristol Children's Hospital, United Bristol Healthcare NHS Trust, Bristol BS3 8BJ

${ }^{7}$ Research and Development Support Unit, Royal Devon and Exeter Foundation NHS Trust, Exeter EX2 5DW

Correspondence to: G Lloyd gavin.lloyd@rdeft.nhs.uk

Cite this as: $B M J$ 2008;337:a2428 doi:10.1136/bmj.a2428

\section{ABSTRACT}

Objective To determine whether full elbow extension as assessed by the elbow extension test can be used in routine clinical practice to rule out bony injury in patients presenting with elbow injury.

Design Adults: multicentre prospective interventional validation study in secondary care. Children: multicentre prospective observational study in secondary care. Setting Five emergency departments in southwest England.

Participants 2127 adults and children presenting to the emergency department with acute elbow injury.

Intervention Elbow extension test during routine care by clinical staff to determine the need for radiography in adults and to guide follow-up in children.

Main outcome measures Presence of elbow fracture on radiograph, or recovery with no indication for further review at 7-10 days.

Results Of 1740 eligible participants, 602 patients were able to fully extend their elbow; 17 of these patients had a fracture. Two adult patients with olecranon fractures needed a change in treatment. In the 1138 patients without full elbow extension, 521 fractures were identified. Overall, the test had sensitivity and specificity (95\% confidence interval) for detecting elbow fracture of $96.8 \%$ (95.0 to 98.2 ) and $48.5 \%$ (45.6 to 51.4). Full elbow extension had a negative predictive value for fracture of $98.4 \%(96.3$ to 99.5$)$ in adults and $95.8 \%$ (92.6 to 97.8$)$ in children. Negative likelihood ratios were 0.03 (0.01 to 0.08 ) in adults and 0.11 (0.06 to 0.19 ) in children.

Conclusion The elbow extension test can be used in routine practice to inform clinical decision making. Patients who cannot fully extend their elbow after injury should be referred for radiography, as they have a nearly $50 \%$ chance of fracture. For those able to fully extend their elbow, radiography can be deferred if the practitioner is confident that an olecranon fracture is not present. Patients who do not undergo radiography should return if symptoms have not resolved within 7-10 days.

\section{INTRODUCTION}

Elbow injuries are common in primary and secondary care, accounting for 2-3\% of emergency department attendances. ${ }^{1}$ Only a minority of patients with such injuries have a fracture, but although clinical decision rules for other limb injuries are well recognised, ${ }^{23}$ no guidelines have been established to indicate which patients with an elbow injury require radiography. An effective clinical decision rule to exclude fracture in acute elbow injury would prevent unnecessary radiography, and could reduce expenditure. ${ }^{4}$

Previous small studies indicate that the ability to fully extend the elbow might rule out clinically significant bony injury. The elbow extension test has therefore been proposed as a simple means of excluding the need for a radiograph, but has yet to be validated in routine practice and has not been well studied in children. ${ }^{5-7}$

Our objective was to determine whether the elbow extension test could be used in routine clinical practice

Box 1 Inclusion and exclusion criteria

Inclusion criteria

- Acute elbow injury

- Adults: age over 15

- Children:

age 3-15

Exclusion criteria

- Previous limited extension

- Altered mental status

- Multiple injuries

- No consent

- No history of trauma

- Injury $>72$ hours old

- Neuromuscular disease

- Suspicion of intentional injury

- Osteogenesis imperfecta 
to rule out bony injury in patients presenting with acute elbow injury.

\section{METHODS}

\section{Design and setting}

We did a multicentre, prospective validation study in adults and an observational study in children who presented with acute elbow injury to five emergency departments in southwest England, UK. As the diagnostic accuracy of the test had not been assessed in children, we did not think that an interventional study was justified in this group. The study was conducted and reported in accord with STARD principles. ${ }^{8} \mathrm{We}$ delivered standardised training for the elbow extension test to emergency nurse practitioners and doctors.

\section{Participants}

Adults ( $>15$ years old) and children (3-15 years) presenting to the participating centres within 72 hours of elbow injury were consecutively recruited to the trials with informed written consent. Box 1 shows inclusion and exclusion criteria.

We judged that for the elbow extension test to be clinically acceptable as a single test for universal use to rule out elbow fracture sensitivity needed to be greater than $99 \%$. With the $3 / \mathrm{n}$ rule for zero numerators, ${ }^{9} 300$ adults and 300 children with full elbow extension and no significant fracture would yield a test sensitivity of $100 \%$ for each group, with $95 \%$ confidence intervals between $99 \%$ and $100 \%$.

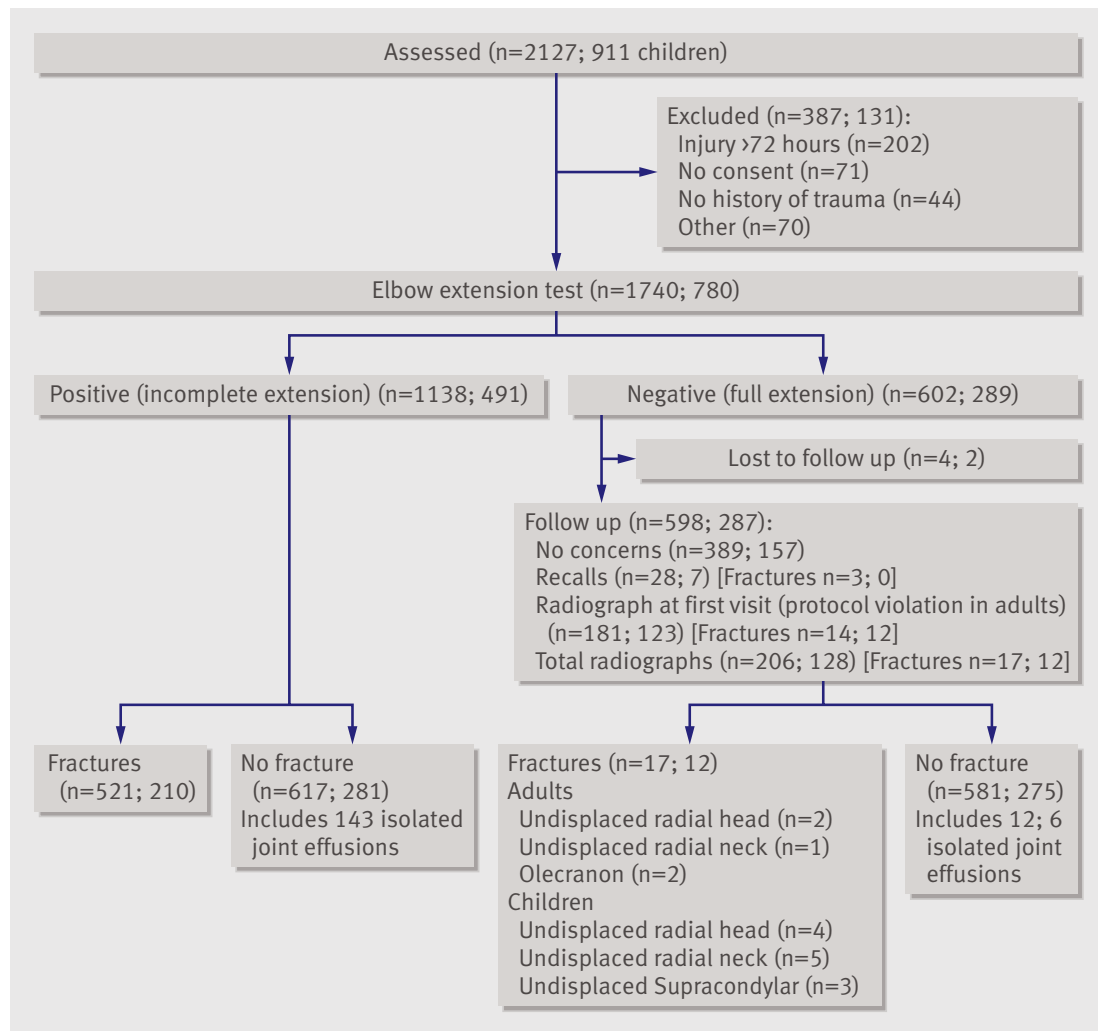

Details of patients undergoing the elbow extension test. Combined totals are shown, with numbers of children in parentheses
Table 1| Details of elbow injuries identified in recruited patients. Values are numbers (percentages)

\begin{tabular}{lcc} 
Type of injury or fracture & Adults & Children \\
Radial head & $204(64)$ & $38(17)$ \\
\hline Olecranon & $28(9)$ & $12(5)$ \\
\hline Radial neck & $18(6)$ & $10(5)$ \\
\hline Supracondylar & $15(5)$ & $106(48)$ \\
\hline Dislocations & $20(6)$ & $5(2)$ \\
\hline Other & $31(10)$ & $51(23)$ \\
\hline Totals & 316 & 222 \\
\hline
\end{tabular}

\section{Interventions}

All patients with elbow injury were identified on arrival during normal registration and triage, and were given analgesia in accord with standard protocols. An emergency department doctor or emergency nurse practitioner then screened and recruited each patient during routine care. A pilot study of this system indicated that $97.9 \%$ of patients presenting with elbow injury were successfully screened. Recruitment rate was monitored and was constant between the centres.

After obtaining consent, the treating practitioner performed the standardised elbow extension test (box 2) as part of the examination. Adult patients with full extension (negative test result) did not undergo radiography and were discharged with analgesia and a sling as needed. Children underwent radiography at the discretion of the treating practitioner, regardless of the result of the elbow extension test. All patients who did not undergo radiography received a structured followup assessment by telephone at 7-10 days. Patients who met any of the recall criteria (box 3) were recalled to the emergency department for radiography. Those not requiring recall were assumed not to have clinically significant bony injury.

The reference standard was the final discharge diagnosis for patients followed up in an orthopaedic clinic, the formal report of a radiologist blinded to the result of the extension test for those not followed up in an orthopaedic clinic, and the result of the structured telephone interview at 7-10 days for those who did not undergo follow-up in an orthopaedic clinic or undergo radiography.

We calculated test characteristics (sensitivity, specificity, predictive values and likelihood ratios) with 95\% confidence intervals, and compared proportions by $\chi^{2}$ test to obtain $\mathrm{P}$ values, using StatsDirect version 2.5.6 (StatsDirect, Altrincham, UK). Binomial proportions were calculated with an exact binomial confidence

\section{Box 2 The elbow extension test}

The seated patient, with exposed and supinated arms, is asked to flex their shoulders to 90 degrees and then fully extend and lock both elbows. Injured and uninjured sides are compared visually and those with equal extension recorded as "full extension." 
Table 2 | Results and outcomes of the elbow extension test

\begin{tabular}{|c|c|c|c|c|c|c|}
\hline & \multicolumn{3}{|c|}{ Adults } & \multicolumn{3}{|c|}{ Children } \\
\hline & No fracture & Fracture & Totals & No fracture & Fracture & Totals \\
\hline $\begin{array}{l}\text { Not full extension } \\
\text { (test positive) }\end{array}$ & $\begin{array}{c}336(84 \\
\left.\text { effusions }{ }^{\star}\right)\end{array}$ & 311 & 647 & $\begin{array}{c}281(59 \\
\left.\text { effusions }{ }^{\star}\right)\end{array}$ & 210 & 491 \\
\hline $\begin{array}{l}\text { Full extension } \\
\text { (test negative) }\end{array}$ & 306 (6 effusions $\left.{ }^{*}\right)$ & 5 & 311 & 275 (6 effusions*) & 12 & 287 \\
\hline Total & 642 & 316 & 958 & 556 & 222 & 778 \\
\hline
\end{tabular}

* Includes isolated effusions with no report or final diagnosis of fracture.

interval, using the Clopper-Pearson method ${ }^{10}$ and for likelihood ratios, we used the Koopman method. ${ }^{11}$

\section{RESULTS}

We screened 2127 patients for eligibility over 21 months (July 2004-April 2006). Of these, 960 adults and 780 children were recruited to the study and underwent the elbow extension test. The age range of the adults was $16-94$ (mean 38 ) years; $51 \%$ were male. Among the children, the age range was 3-15 (mean 10) years and 52\% were male. The overall prevalence of fracture was $31 \%(538 / 1740$, table 1$)$. We summarise recruitment and results of the test in the figure and table 2 .

\section{Adults}

Of the 958 adults included in the analysis, $313(33 \%)$ were able to fully extend their elbow, and of these patients all but two were followed up. Five fractures were identified in those patients with full elbow extension, and of these, two required operative intervention (both olecranon fractures).

Seven hundred and five adults (73\%) underwent radiography at their first visit. Fifty eight protocol violations occurred, mostly when temporary staff misunderstood or were unaware of the protocol (52 patients), but also in patients who underwent radiography for a potential foreign body (three) or at the request of their general practitioner (three).

Of the 647 adults who could not fully extend their injured elbow, $311(48 \%)$ had confirmed fractures and 84 had elbow joint effusions.

\section{Children}

Of the 778 children included in the analysis, 289 (37\%) could fully extend their elbow, and of these patients all but two were followed up. We found 12 fractures (all identified at first visit) and six effusions in those with full elbow extension, none of which required operative intervention.

Of the 491 children who could not fully extend their injured elbow, 210 (43\%) had confirmed fractures and 59 had elbow joint effusions.

\section{Test characteristics}

A reference standard was determined in 1736 of the 1740 patients. Test characteristics are shown in table 3 . Overall, test sensitivity for detecting elbow fracture was $96.8 \%$ (95\% confidence interval 95.0 to 98.2$)$ and specificity was $48.5 \%$ (45.6 to 51.4 ). A "worst case" sensitivity analysis, assuming that fractures were present in the four patients who were lost to followup and in all patients with effusions, gave an overall sensitivity of $95.3 \%$ for the detection of fracture.

For adult patients with full elbow extension, the test had a negative predictive value for fracture of $98.4 \%$ (95\% confidence interval 96.3 to 99.5$)$ and negative likelihood ratio of 0.03 ( 0.01 to 0.08$)$. In children the negative predictive value for fracture was $95.8 \%$ (92.6 to 97.8 ) and negative likelihood ratio 0.11 (0.06 to $0.19)$.

In practice, therefore, adults who could fully extend their elbow after acute injury had a $1.6 \% \quad(95 \%$ confidence interval 0.5 to 3.7$)$ chance of fracture. In children the risk was $4.2 \%$ (2.2 to 7.4 ), despite the greater prevalence of fracture in adults $(316 / 958,33 \%)$ than in children $\left(222 / 778,29 \%: \chi^{2}=3.98, \mathrm{P}=0.046\right.$, $\mathrm{df}=1$ ). The proportion of patients with a fracture who were not able to fully extend their elbow (sensitivity) was significantly greater in adults $(311 / 316,98.4 \%)$ than in children $\left(210 / 222,94.6 \%: \chi^{2}=6.23, \mathrm{P}=0.013\right.$, $\mathrm{df}=1)$. The specificity of the test did not differ between adults $(306 / 642,47.7 \%)$ and children $(275 / 556,49.5 \%$ : $\left.\chi^{2}=0.39, \mathrm{P}=0.53, \mathrm{df}=1\right)$.

\section{DISCUSSION}

In this study we found that the elbow extension test, used in routine clinical practice, has a high sensitivity and negative predictive value for elbow fracture. The test was able to rule out a fracture and the need for radiography in about a quarter of patients presenting with acute elbow injury. This finding is useful, as over a third of patients with elbow injury ${ }^{5-7}$ are able to fully extend their elbow at presentation. Patients who could not fully extend their elbow had a nearly $50 \%$ chance of radiologically confirmed fracture.

Table 3 | Elbow extension test characteristics (95\% confidence intervals shown in parentheses)

\begin{tabular}{|c|c|c|c|c|c|c|}
\hline & \multicolumn{2}{|c|}{ Adults } & \multicolumn{2}{|c|}{ Children } & \multicolumn{2}{|c|}{ Combined } \\
\hline & Fracture & Fracture or effusion & Fracture & Fracture or effusion & Fracture & Fracture or effusion \\
\hline Sensitivity & $98.4(96.3$ to 99.5$)$ & 97.3 (95.2 to 98.6$)$ & 94.6 (90.7 to 97.2 ) & 93.7 (90.3 to 96.2) & 96.8 (95.0 to 98.2$)$ & 95.8 (94.0 to 97.2$)$ \\
\hline Specificity & 47.7 (43.7 to 51.6$)$ & $54.3(50.1$ to 58.6$)$ & 49.5 (45.2 to 53.7$)$ & 54.8 (50.3 to 59.2$)$ & $48.5(45.6$ to 51.4$)$ & $54.6(51.5$ to 57.6$)$ \\
\hline Negative predictive value & 98.4 (96.3 to 99.5$)$ & 96.5 (93.8 to 98.2) & 95.8 (92.6 to 97.8$)$ & 93.7 (90.1 to 96.2) & 97.2 (95.5 to 98.3$)$ & 95.2 (93.1 to 96.7$)$ \\
\hline Positive predictive value & 48.1 (44.2 to 52.0$)$ & $61.0(57.2$ to 64.8$)$ & 42.8 (38.4 to 47.3$)$ & $54.8(50.3$ to 59.2$)$ & 45.8 (42.9 to 48.7$)$ & 58.3 (55.4 to 61.2$)$ \\
\hline Positive likelihood ratio & $1.88(1.75$ to 2.03$)$ & 2.13 (1.95 to 2.34$)$ & 1.87 (1.72 to 2.05$)$ & 2.07 (1.88 to 2.30$)$ & 1.88 (1.78 to 1.99$)$ & 2.11 (1.97 to 2.26$)$ \\
\hline Negative likelihood ratio & 0.03 (0.01 to 0.08$)$ & 0.05 (0.03 to 0.09$)$ & 0.11 (0.06 to 0.19$)$ & $0.11(0.07$ to 0.18$)$ & $0.06(0.04$ to 0.10$)$ & 0.08 (0.05 to 0.11$)$ \\
\hline
\end{tabular}


Box 3 Criteria for recall

- Inability to fully straighten elbow

- Pain worsening or not improving

- Any functional problems (any difficulty using arm)

- Any concern of the patient or researcher not covered by the above

The low negative likelihood ratio of 0.03 confirms that this is a powerful test to rule out fracture in adults, ${ }^{12}$ but the test does not exceed the sensitivity of $99 \%$ that we had previously judged as being clinically desirable. Ninety nine per cent sensitivity is a challenging standard, and our test has similar properties, in terms of sensitivity and specificity, to established clinical decision rules for other joints. ${ }^{13}$ Ultimately, application of this test will rely on physicians' judgment, informed by the risk and consequences of false negatives, and by the availability of a gold standard diagnostic test (radiography) and follow-up. Most false negative results are likely to be minor or occult fractures that require no change in treatment. ${ }^{14}$ However, we advise caution in the use of the elbow extension test as a single clinical decision rule for universal use, in view of the two olecranon fractures in adults, and the risk of occult supracondylar fractures in children. ${ }^{15}$ The false negative rate is also higher in children than adults.

\section{STRENGTHS AND LIMITATIONS}

The strengths of this study were that the elbow extension test was carried out by usual practitioners in the emergency department during routine assessment of patients, reflecting the probable application of this test in real practise. The sample size was sufficient to meet our objectives, with suitably narrow confidence intervals. A high follow-up rate was essential to the study design, and ensured that a sensitivity analysis made no significant difference to the results.

It is possible that our follow-up protocol might not have identified all patients with a fracture undetected by the test, and the recall criteria used are not validated. However, significant injuries are unlikely to have been missed using this low threshold for patient recall, and a

\section{WHAT IS ALREADY KNOWN ON THIS TOPIC}

No clinical decision rule exists for deciding which patients with acute elbow injury require radiography

The elbow extension test has been proposed as a simple test to rule out the need for radiography, but it has not been validated in routine practice

\section{WHAT THIS STUDY ADDS}

The elbow extension test can be used in routine practice

The test effectively rules out the need for radiography in patients with a recent elbow injury and full joint extension; caution should be used in children and in patients with suspected olecranon fracture review of the database found no evidence of subsequent reattendance in patients who were discharged.

We did not assess interobserver agreement, and there was no mechanism to record or analyse equivocal results. While this may have contributed to the worse performance of the test in children than in adults, an under appreciation of the normal hyperextension in some children's elbows, or inadequate comparisons to the uninjured limb, are other possible explanations.

\section{COMPARISON WITH PREVIOUS STUDIES}

The incidences of full elbow extension and fracture in our study were similar to those reported in previous smaller studies. ${ }^{156}$ The sensitivity of the test was also consistent with these studies, but with much narrower confidence intervals. Lennon et al recommended testing a full range of all elbow movements (extension, flexion, and supination) to exclude the need for radio graphy. ${ }^{1}$ However, although they report a sensitivity of $97.6 \%$, similar to that seen in our study, they excluded patients "not requiring an $x$ ray", and the reduced specificity of $21 \%$ undermines the value of this approach in practice. This more complicated test therefore seems to have no advantage over testing full extension alone.

Modifying the elbow extension test in an attempt to improve sensitivity would probably undermine its specificity and clinical usefulness. Elbow extension alone is a highly sensitive test, is effective in routine practice, and can usefully inform clinical decision making.

\section{CONCLUSIONS}

We conclude that patients with recent elbow injury who cannot fully extend their elbow should be referred for radiography. Those who are able to fully extend do not need radiography, provided the practitioner is confident that olecranon fracture is not present, that caution is used in children, and that the patient can return for reassessment if their symptoms have not resolved in 7-10 days.

We thank Beth Newstead, Charlotte Pagram, Julie Small and the reception and clinical staff of participating hospitals for their assistance and support of this study.

Contributors: AA and ADR co-wrote the manuscript. AA, ADR, JRB, and G formed the SWEET research committee, which designed and

implemented the study, and reviewed the manuscript. GL is the guarantor. FB, JD, SH, IH, JAK, SLeR, SSMS, RT, and JV reviewed the manuscript and CO-ordinated the trial at their respective centres.

Funding: Research grant from the College of Emergency Medicine. The research was independent of the funders.

Competing interests: None declared.

Ethical approval: Each site obtained approval from local ethics committee All eligible patients were recruited after written informed consent had been obtained

Provenance and peer review: Not commissioned; externally peer reviewed.

1 Lennon RI, Riyat MS, Hilliam R, Anathkrishnan G, Alderson G. Can a normal range of elbow movement predict a normal elbow $x$ ray? Emerg Med J 2007;24:86-8

2 Stiell IG, Greenberg GH, McKnight RD, Nair RC, McDowell I, Reardon M, et al. Decision rules for the use of radiography in acute ankle injuries. Refinement and prospective validation. JAMA 1993;269:1127-32. 
3 Stiell IG, Wells GA, McDowell I, Greenberg GH, McKnight RD, Cwinn AA, et al. Use of radiography in acute knee injuries: need for clinical decision rules. Acad Emerg Med 1995;2:966-73.

4 Perry JJ, Stiell IG. Impact of clinical decision rules on clinical care of traumatic injuries to the foot and ankle, knee, cervical spine, and head. Injury 2006;37:1157-65.

5 Docherty MA, Schwab RA, Ma OJ. Can elbow extension be used as a test of clinically significant injury? South Med / 2002;95:539-41.

6 Hawksworth CR, Freeland P. Inability to fully extend the injured elbow: an indicator of significant injury. Arch Emerg Med 1991;8:253-6.

7 Dildar S. Inability to fully extend and supinate the injured elbow: an indicator of significant injury. Todays Emergency 2007;12:6-7.

8 Bossuyt PM, Reitsma JB, Bruns DE, Gatsonis CA, Glasziou PP, Irwig LM, et al. Towards complete and accurate reporting of studies of diagnostic accuracy: The STARD initiative. Clin Chem 2003;49:1-6.

9 Hanley JA, Lippman-Hand A. If nothing goes wrong, is everything alright? JAMA 1983;249:1743-5.
10 Zhou X, Obuchowski NA, McClish DK. Statistical Methods in Diagnostic Medicine New York: Wiley, 2002.

11 Koopman PAR. Confidence limits for the ratio of two binomial proportions. Biometrics 1984;40:513-7.

12 Worster A, Innes G, Abu-Laban RB. Diagnostic testing: an emergency medicine perspective. CJEM 2002;4:348-54.

13 Bachmann LM, Kolb E, Koller MT, Steurer J, Riet G. Accuracy of Ottawa ankle rules to exclude fractures of the ankle and mid-foot: systematic review. BMJ 2003;326:417-23.

14 Gorzack A, Mackway-Jones K. Repeat radiography is not needed for traumatic elbow effusions with no fracture on initial x-ray. Best Bets Topics, 1999 (modified 2003). www.bestbets.org.uk.

15 Griffith JF, Roebuck DJ, Cheng JC, Chan YL, RainerTH, Ng BK, et al. Acute elbow trauma in children: spectrum of injury revealed by MR imagin not apparent on radiographs. Am J Roentgenol 2001;176:53-60.

Accepted: 15 September 2008 